PROCEEDINGS OF THE

AMERICAN MATHEMATICAL SOCIETY

Volume 125, Number 7, July 1997, Pages 1921-1931

S $0002-9939(97) 03814-8$

\title{
INVARIANTS OF SOME ABELIAN $p$-GROUPS IN CHARACTERISTIC $p$
}

\author{
MARA D. NEUSEL
}

(Communicated by Wolmer V. Vasconcelos)

\begin{abstract}
An explicit description of the ring of polynomial invariants of cyclic groups of order $p^{s}$ with fixed point set of codimension 2, or with covariants of codimension 2 , over a field of characteristic $p$ is given. It transpires that these rings are complete intersections. A slight generalization for some abelian $p$-groups is also derived, which leads to a result about arbitrary groups with $p$-Sylow subgroup of this type.
\end{abstract}

\section{INTRODUCTION}

Let $\mathbb{F}$ be a finite field of characteristic $p \neq 0$. Let $\rho: G \hookrightarrow \operatorname{GL}(n, \mathbb{F})$ be a faithful representation of a finite group $G$, where $p$ divides the order of $G,|G|$. The group $G$ acts on $\mathrm{V}=\mathbb{F}^{n}$, the $n$-dimensional vector space over $\mathbb{F}$. Denote by $\rho^{*}$ the dual representation of $G$ on $\mathrm{V}^{*}$ and $x_{1}, \ldots, x_{n}$ the standard dual basis. Then $\rho$ induces an action of $G$ on the ring of polynomials $\mathbb{F}[\mathrm{V}]=\mathbb{F}\left[x_{1}, \ldots, x_{n}\right]$. Define the vector space of covariants

$$
\mathrm{V}_{G}:=\mathrm{V} / \operatorname{span}_{\mathbb{F}}\{g v-v \mid g \in G, v \in \mathrm{V}\} \cong \operatorname{Hom}_{\mathbb{F}}\left(\left(\mathrm{V}^{*}\right)^{G}, \mathbb{F}\right),
$$

which is dual to the fixed point set $\left(\mathrm{V}^{*}\right)^{G}$. By a result of Nakajima [6] and Landweber-Stong [4], or [7], section 8.2, it is known that for groups $G$ with either $\mathrm{V}^{G}$ or $\mathrm{V}_{G}$ of codimension 1 , both rings of invariants $\mathbb{F}[\mathrm{V}]^{G}$ and $\mathbb{F}\left[\mathrm{V}^{*}\right]^{G}$ are polynomial. I am going to show that a similar result holds for the case of $\operatorname{codim}_{\mathbb{F}}\left(\mathrm{V}^{G}\right)=2$ or $\operatorname{codim}_{\mathbb{F}}\left(\mathrm{V}_{G}\right)=2$, and $G$ a cyclic group of order $p^{s}$ : both rings of invariants $\mathbb{F}[\mathrm{V}]^{G}$ and $\mathbb{F}\left[\mathrm{V}^{*}\right]^{G}$ are complete intersections. This sharpens a result of EllingsrudSkjelbred [2]. They have shown that for cyclic groups of prime power order the ring of polynomial invariants is Cohen-Macaulay, if the codimension of the fixed point set is 2, and has homological codimension less than the Krull dimension, if the codimension of the fixed point set is greater than $2 .{ }^{1}$

These results can be generalized to some abelian $p$-groups.

The method used to derive these results seems to be new. It consists of four steps.

Received by the editors October 23, 1995 and, in revised form, January 16, 1996

1991 Mathematics Subject Classification. Primary 13A50.

Key words and phrases. Polynomial invariants of finite groups.

${ }^{1}$ Hence the results of Ellingsrud-Skjelbred and Landweber-Stong show that $\mathbb{F}[\mathrm{V}]^{\mathbb{Z} / p^{s}}$ is CohenMacaulay if and only if $\operatorname{codim}_{\mathbb{F}}\left(\mathrm{V}^{\mathbb{Z} / p^{s}}\right) \leq 2$ or $\operatorname{codim}_{\mathbb{F}}\left(\mathrm{V}_{\mathbb{Z} / p^{s}}\right) \leq 2$, because $\operatorname{codim}_{\mathbb{F}}\left(\mathrm{V}^{\mathbb{Z} / p^{s}}\right)=$ $\operatorname{codim}_{\mathbb{F}}\left(\mathrm{V}_{\mathbb{Z} / p^{s}}\right)$. 
(1) The first step is to construct a model ring $A$, which in the end will turn out to be the desired ring of invariants. The model is a subalgebra of $\mathbb{F}[\mathrm{V}]^{G}$, and the extension $A \subseteq \mathbb{F}[\mathrm{V}]^{G}$ is finite.

(2) I compute the degree ([7], section 5.5), $\operatorname{deg}(A)$, of $A$ from its Poincaré series, and then use the degree theorem ([7], Theorem 5.5.3) to conclude that $A$ and $\mathbb{F}[\mathrm{V}]^{G}$ have the same fields of fractions.

(3) Extending scalars from $\mathbb{F}$ to a larger field $\mathbb{K}$ I am able to show that $\mathbb{K} \otimes_{\mathbb{F}} A$ is a unique factorization domain, and hence by a theorem ${ }^{2}$ of Nagata [5] that $A$ itself is a unique factorization domain and therefore integrally closed.

(4) Since $A \subseteq \mathbb{F}[\mathrm{V}]^{G}$ is an integral extension, and both $A$ and $\mathbb{F}[\mathrm{V}]^{G}$ have the same field of fractions, it follows that $A=\mathbb{F}[\mathrm{V}]^{G}$, i.e. I have shown that the model ring is the ring of invariants.

In the first part of this paper, I am going to handle the case of cyclic groups of prime order $p$. The second part shows that for $p$ odd these are the only such examples, handles the case $p=2$, and derives some corollaries for arbitrary finite groups.

As a general reference for invariant theory of finite groups I recommend [7].

This work was done during my stay at the Mathematics Department of Yale University in spring term 1995. I want to thank the members of the department for the possibility to work at their institute and to enjoy the warm and friendly atmosphere. I thank Larry Smith for his encouragement and help.

\section{CyClic Groups OF PRIME ORDER}

Theorem 2.1. Suppose $\mathbb{F}$ is a field of characteristic $p$ and $\mathrm{V}=\mathbb{F}^{n}$. Let $\rho: G=$ $\mathbb{Z} / p \hookrightarrow \mathrm{GL}(n, \mathbb{F})$ be a faithful representation of a cyclic group of prime order $p$, and let $\operatorname{codim}_{\mathbb{F}}\left(\mathrm{V}^{G}\right)=2$. Then the ring of polynomial invariants is a complete intersection, more precisely

$$
\mathbb{F}[\mathrm{V}]^{\mathbb{Z} / p}=\mathbb{F}\left[l_{1}, \ldots, l_{n-2}, q, c_{p, 1}, c_{p, 2}\right] /\left(r_{2 p}\right),
$$

where $\operatorname{deg}\left(l_{i}\right)=1, i=1, \ldots, n-2, \operatorname{deg}(q)=2, \operatorname{deg}\left(c_{p, j}\right)=p, j=1,2, \operatorname{deg}\left(r_{2 p}\right)=$ $2 p$. Moreover

$$
\mathbb{F}\left[\mathrm{V}^{*}\right]^{\mathbb{Z} / p}=\mathbb{F}\left[x_{1}, \ldots, x_{n-2}, q^{\prime}, c_{p, n-1}^{\prime}, c_{p, n}^{\prime}\right] /\left(r_{2 p}^{\prime}\right)
$$

is also a complete intersection, where $\operatorname{deg}\left(q^{\prime}\right)=2, \operatorname{deg}\left(c_{p, j}^{\prime}\right)=p, j=n-1, n$, $\operatorname{deg}\left(r_{2 p}^{\prime}\right)=2 p$.

For the proof I need the following lemma:

Lemma 2.2. Let $A=\mathbb{K}\left[f_{1}, \ldots, f_{m}\right] /\left(r_{1}, \ldots, r_{l}\right)$ be a complete intersection with Krull dimension $\operatorname{dim} A=n$ where $\mathbb{K}$ is any field. Then ${ }^{3}$

$$
\operatorname{deg}(A)=\frac{\prod_{j=1}^{l} \operatorname{deg}\left(r_{j}\right)}{\prod_{i=1}^{m} \operatorname{deg}\left(f_{i}\right)}
$$

\footnotetext{
${ }^{2}$ Theorem (Nagata). Let $\mathbb{K}$ be a field. Let I be a homogeneous ideal of $\mathbb{K}\left[x_{1}, \ldots, x_{n}\right]$. If there exists a field $\mathbb{K}^{\prime}$ containing $\mathbb{K}$ such that $\mathbb{K}^{\prime}\left[x_{1}, \ldots, x_{n}\right] / I$ is a unique factorization ring, then $\mathbb{K}\left[x_{1}, \ldots, x_{n}\right] / I$ is also a unique factorization ring.

${ }^{3}$ For a definition of the degree of a graded algebra see [7], section 5.5.
} 
Proof. Since $A$ is a complete intersection, $\left(r_{1}, \ldots, r_{l}\right)$ is a regular sequence, and $m-l=n$. Hence the Poincaré series of $A$ is given by

$$
\mathcal{P}(A, t)=\frac{\prod_{j=1}^{l}\left(1-t^{\operatorname{deg}\left(r_{j}\right)}\right)}{\prod_{i=1}^{m}\left(1-t^{\operatorname{deg}\left(f_{i}\right)}\right)}=\left(\frac{1}{(1-t)^{n}}\right) \frac{\prod_{j=1}^{l}\left(1+t+\ldots+t^{\operatorname{deg}\left(r_{j}\right)-1}\right)}{\prod_{i=1}^{m}\left(1+t+\cdots+t^{\operatorname{deg}\left(f_{i}\right)-1}\right)} .
$$

Therefore since $\operatorname{deg}(A)=\left.(1-t)^{n} \mathcal{P}(A, t)\right|_{t=1}$ the result follows.

Remark 1. If $A=\mathbb{K}\left[f_{1}, \ldots, f_{m}\right] /\left(r_{1}, \ldots, r_{l}\right)=\mathbb{K}[\mathrm{V}]^{G}$ is the ring of polynomial invariants for some faithful representation $\rho: G \hookrightarrow \mathrm{GL}(n, \mathbb{K})$ of a finite group $G$, then this lemma leads to the formula

$$
\frac{\prod_{i=1}^{m} \operatorname{deg}\left(f_{i}\right)}{\prod_{j=1}^{l} \operatorname{deg}\left(r_{j}\right)}=\frac{1}{\operatorname{deg}(A)}=|G|,
$$

by [7], Theorem 5.5.3.

Remark 2. This lemma is sharp, in the sense that if $A=\mathbb{K}\left[f_{1}, \ldots, f_{m}\right] /\left(r_{1}, \ldots, r_{l}\right)$ is only Cohen-Macaulay this formula is not true: consider the representation $\rho$ : $\mathbb{Z} / k \hookrightarrow \mathrm{GL}(n, \mathbb{C})$ given by the matrix

$$
T=\left(\begin{array}{cc}
\exp (2 \pi i / k) & 0 \\
0 & \exp (2 \pi i / k)
\end{array}\right)
$$

for an integer $k$. Then the ring of polynomial invariants is

$$
\mathbb{C}[x, y]^{\mathbb{Z} / k}=\mathbb{C}\left[f_{0}, \ldots, f_{k}\right] /\left(f_{0} f_{k}-f_{1} f_{k-1}, \ldots, f_{0} f_{k}-f_{\alpha} f_{k-\alpha}\right),
$$

where $f_{i}=x^{i} y^{k-i}, i=0, \ldots, k$, and $\alpha=\frac{k-1}{2}$, resp. $\frac{k}{2}$ for $k \equiv 1$, resp. $0 \bmod 2$. Obviously

$$
\frac{\prod_{i=0}^{k} \operatorname{deg}\left(f_{i}\right)}{\prod_{i=1}^{\alpha} \operatorname{deg}\left(f_{0} f_{k}-f_{i} f_{k-i}\right)}=\frac{\left(k^{k+1}\right)}{(2 k)^{\alpha}} \neq k, \quad \text { for } k \geq 3 .
$$

By a theorem of Eagon-Hochster the ring of invariants, $\mathbb{C}[\mathrm{V}]^{\mathbb{Z} / k}$, is Cohen-Macaulay [7], Theorem 6.7.8.

Proof of the theorem. Since $\operatorname{codim}_{\mathbb{F}}\left(\mathrm{V}^{\mathbb{Z} / p}\right)=2$, the image of a generator of $\mathbb{Z} / p$ under the representation $\rho$ has the form

$$
T=\left(\begin{array}{cc}
I & B \\
0 & M
\end{array}\right)
$$

where $M \in \mathrm{GL}(2, \mathbb{F}), B \in M((n-2) \times 2, \mathbb{F})$ is an $(n-2) \times 2$-matrix with entries in $\mathbb{F}, I=\mathrm{id} \in \mathrm{GL}(n-2, \mathbb{F})$, and ${ }^{4}$

$$
\begin{aligned}
& M^{p}=\mathrm{id} \text { and } B+B M+\cdots+B M^{p-1}=0, \\
& M^{p-1} \neq \mathrm{id} \text { or } B+B M+\cdots+B M^{p-2} \neq 0 .
\end{aligned}
$$

The dual representation $\rho^{*}$ maps a generator of $\mathbb{Z} / p$ to the transposed matrix, and similarly the relations are transposed.

I am going to distinguish two cases.

Case 1: $M=$ id.

$$
{ }^{4} T^{k}=\left(\begin{array}{cc}
I & B+B M+\cdots+B M^{k-1} \\
0 & M^{k}
\end{array}\right), \text { so the condition that follows is what is needed for } T
$$

to have order $p$. 
First I consider the case of the representation $\rho$. If $M=$ id, then

$$
B=\left(\begin{array}{cc}
b_{1,1} & b_{1,2} \\
\vdots & \vdots \\
b_{n-2,1} & b_{n-2,2}
\end{array}\right) \in M((n-2) \times 2, \mathbb{F}),
$$

with $\operatorname{rk}(B)=2, n \geq 4$, and without loss of generality I assume that the first two rows are linearly independent. Let

$$
A:=\mathbb{F}\left[l_{3}, \ldots, l_{n-2}, x_{n-1}, x_{n}, q, c_{p}\left(x_{1}\right), c_{p}\left(x_{2}\right)\right] /\left(r_{2 p}\right),
$$

where $c_{p}\left(x_{1}\right)$, resp. $c_{p}\left(x_{2}\right)$, are the top orbit Chern classes of $x_{1}$, resp. $x_{2}$ (see [7], section 3.2 for a discussion of orbit Chern classes),

$$
q=x_{1}\left(b_{2,1} x_{n-1}+b_{2,2} x_{n}\right)-x_{2}\left(b_{1,1} x_{n-1}+b_{1,2} x_{n}\right)
$$

is a generator of degree 2 ,

$$
l_{i}=\left(b_{2,1} b_{1,2}-b_{1,1} b_{2,2}\right)\left(b_{2,1} x_{i}-b_{i, 1} x_{2}\right)-\left(b_{i, 2} b_{2,1}-b_{i, 1} b_{2,2}\right)\left(b_{2,1} x_{1}-b_{1,1} x_{2}\right)
$$

are linear generators for $i=3, \ldots, n-2$ and

$$
\begin{aligned}
r_{2 p}= & c_{p}\left(x_{1}\right)\left(b_{2,1} x_{n-1}+b_{2,2} x_{n}\right)^{p}-c_{p}\left(x_{2}\right)\left(b_{1,1} x_{n-1}+b_{1,2} x_{n}\right)^{p}-q^{p} \\
& +q\left(b_{1,1} x_{n-1}+b_{1,2} x_{n}\right)^{p-1}\left(b_{2,1} x_{n-1}+b_{2,2} x_{n}\right)^{p-1}
\end{aligned}
$$

is a relation of degree $2 p$ satisfied by these polynomials. First, I am going to show that $A \subset \mathbb{F}[\mathrm{V}]^{\mathbb{Z} / p}$ : Since $A$ is generated by polynomials, which are invariant under $\mathbb{Z} / p$, and $r_{2 p}$ is a relation satisfied by these, it follows that there is a map of algebras

$$
\psi: A \rightarrow \mathbb{F}[\mathrm{V}]^{\mathbb{Z} / p} .
$$

Since $\mathbb{F}[\mathrm{V}]^{\mathbb{Z} / p} \subset \mathbb{F}[\mathrm{V}]$ it is equivalent to show that the composition

$$
\Psi: A \rightarrow \mathbb{F}[\mathrm{V}]^{\mathbb{Z} / p} \hookrightarrow \mathbb{F}[\mathrm{V}]
$$

is monic. This is equivalent to showing that $\left(r_{2 p}\right)$ is the kernel of

$$
\varphi: A^{\prime}:=\mathbb{F}\left[l_{3}, \ldots, l_{n-2}, x_{n-1}, x_{n}, q, c_{p}\left(x_{1}\right), c_{p}\left(x_{2}\right)\right] \rightarrow \mathbb{F}[\mathrm{V}] .
$$

Since $\mathbb{F}[\mathrm{V}]$ is an integral domain, $(0) \subset \mathbb{F}[\mathrm{V}]$ is a prime ideal, hence $\operatorname{ker}(\varphi) \subset A^{\prime}$ is a prime ideal, which contains $r_{2 p}$, hence $\left(r_{2 p}\right) \subset \operatorname{ker}(\varphi)$.

The image of $\varphi$ has Krull dimension $n=\operatorname{dim} \mathbb{F}[\mathrm{V}]$, because it contains a system of parameters, namely ${ }^{5}$

$$
l_{3}, \ldots, l_{n-2}, x_{n-1}, x_{n}, c_{p}\left(x_{1}\right), c_{p}\left(x_{2}\right) .
$$

Therefore the kernel has height 1 . This means that $\operatorname{ker}(\varphi)$ is a prime ideal lying minimally over $\left(r_{2 p}\right)$. In a unique factorization domain, such as $A^{\prime}$, an isolated prime of a principal ideal is itself principal, i.e.

$$
\left(r_{2 p}\right) \subset \operatorname{ker}(\varphi)=(h),
$$

for some $h \in A^{\prime}$ (see e.g. [1], Proposition $3.11 \mathrm{~b}$ ). Therefore $h \mid r_{2 p}$, and $h$ is a relation of degree $\leq \operatorname{deg}\left(r_{2 p}\right)=2 p$. Since $r_{2 p}$ is a relation of minimal degree between $l_{3}, \ldots, l_{n-2}, x_{n-1}, x_{n}, q, c_{p}\left(x_{1}\right), c_{p}\left(x_{2}\right)$, it follows that $h=r_{2 p}$ and hence $\operatorname{ker}(\varphi)=(h)=\left(r_{2 p}\right)$. So, $A \subset \mathbb{F}[\mathrm{V}]$.

\footnotetext{
${ }^{5} l_{3}, \ldots, l_{n-2}, x_{n-1}, x_{n}$ span a subspace complementary to $x_{1}, x_{2}$ and $c_{p}\left(x_{1}\right), c_{p}\left(x_{2}\right)$ are monic polynomials of degree $p$ in $x_{1}$, resp. $x_{2}$ with coefficients in $l_{3}, \ldots, l_{n-2}, x_{n-1}, x_{n}$.
} 
Since $A$ is a complete intersection, it follows from the above Lemma 2.2 that

$$
\operatorname{deg}(A)=\left.(1-t)^{n} \mathcal{P}(A, t)\right|_{t=1}=\frac{2 p}{2 p^{2}}=\frac{1}{p}=\frac{1}{|G|}=\operatorname{deg}\left(\mathbb{F}[\mathrm{V}]^{G}\right) .
$$

So from the degree theorem (loc. cit.) the fields of fractions of both rings are equal, i.e.,

$$
F(A)=F\left(\mathbb{F}[\mathrm{V}]^{\mathbb{Z} / p}\right) .
$$

Define $\mathbb{K}:=\mathbb{F}\left[l_{3}, \ldots, l_{n-2}, x_{n-1}, x_{n}, q, l_{3}^{-1}, \ldots, l_{n-2}^{-1}, x_{n-1}^{-1}, x_{n}^{-1}, q^{-1}\right]$; then

$$
A\left[l_{3}^{-1}, \ldots, l_{n-2}^{-1}, x_{n-1}^{-1}, x_{n}^{-1}, q^{-1}\right]=\mathbb{K}\left[c_{p}\left(x_{1}\right), c_{p}\left(x_{2}\right)\right] /\left(r_{2 p}\right)=\mathbb{K}\left[c_{p}\left(x_{1}\right)\right],
$$

where the last equality holds, because $r_{2 p}$ has become a linear relation (over $\mathbb{K}$ ) between the two generators $c_{p}\left(x_{1}\right), c_{p}\left(x_{2}\right)$. Since a polynomial ring is a unique factorization domain, $A$ is also a unique factorization domain by Nagata's theorem (loc. cit.) so normal.

The extension $A \subset \mathbb{F}[\mathrm{V}]^{\mathbb{Z} / p}$ is integral, both rings are normal, so they are equal.

In the case of the dual representation $\rho^{*}$, define

$$
A:=\mathbb{F}\left[x_{1}, \ldots, x_{n-2}, q^{\prime}, c_{p}^{\prime}\left(x_{n-1}\right), c_{p}^{\prime}\left(x_{n}\right)\right] /\left(r_{2 p}^{\prime}\right),
$$

where $c_{p}^{\prime}\left(x_{n-1}\right)$, resp. $c_{p}^{\prime}\left(x_{n}\right)$, are the top orbit Chern classes of $x_{n-1}$, resp. $x_{n}$,

$$
q^{\prime}=x_{n-1} f_{2}-x_{n} f_{1}
$$

is of degree 2 , where

$$
f_{i}:=\left(\sum_{i=1}^{n-2} b_{i, 1} x_{i}\right) \quad \text { and } \quad f_{2}:=\left(\sum_{i=1}^{n-2} b_{i, 2} x_{i}\right),
$$

and

$$
r_{2 p}^{\prime}=c_{p}^{\prime}\left(x_{n-1}\right) f_{2}^{p}-c_{p}^{\prime}\left(x_{n}\right) f_{1}^{p}-q^{\prime p}+q^{\prime} f_{1}^{p-1} f_{2}^{p-1}
$$

is a relation satisfied by these polynomials. $A=\mathbb{F}\left[\mathrm{V}^{*}\right]^{G}$ follows in the same way as above.

Case 2: $M \neq$ id.

If $M \neq \mathrm{id}$, then $p \neq 2$ and $M \in \operatorname{Syl}_{p}(\mathrm{GL}(2, \mathbb{F}))$, so without loss of generality I assume that $M$ has the form

$$
M=\left(\begin{array}{ll}
1 & 1 \\
0 & 1
\end{array}\right)
$$

Since $\operatorname{codim}_{\mathbb{F}}\left(\mathrm{V}^{\mathbb{Z} / p}\right)=2$ by assumption, it follows that without loss of generality $b_{1,1} \neq 0 \in \mathbb{F}$. Define the model ring $A$ by

$$
A:=\mathbb{F}\left[l_{2}, \ldots, l_{n-2}, x_{n}, q, c_{p}\left(x_{1}\right), c_{p}\left(x_{n-1}\right)\right] /\left(r_{2 p}\right),
$$

where $c_{p}\left(x_{1}\right)$, resp. $c_{p}\left(x_{n-1}\right)$, are the top orbit Chern classes of $x_{1}$, resp. $x_{n-1}$,

$$
q=2 x_{1} x_{n}+\left(b_{1,1}-2 b_{1,2}\right) x_{n-1} x_{n}-b_{1,1} x_{n-1}^{2}
$$

is a generator of degree 2 ,

$$
l_{i}=b_{1,1} x_{i}-b_{i, 1} x_{1}-\left(b_{1,1} b_{i, 2}-b_{i, 1} b_{1,2}\right) x_{n-1}
$$


are linear generators for $i=2, \ldots, n-2$ and

$$
\begin{aligned}
r_{2 p}= & 2 c_{p}\left(x_{1}\right) x_{n}^{p}-q^{p}-b_{1,1} c_{p}\left(x_{n-1}\right)^{2}-\sum_{k=1}^{p-1}\left((-1)^{k} b_{1,1}^{k} a^{2 k} x_{n}^{2 k} q^{p-k}\right) \\
& -\sum_{k=(p-1) / 2}^{p-1} b_{1,1}^{k}\left((-1)^{k}\left(\frac{p-1}{2}\right)^{-1} a^{2 k-(p-1)} x_{n}^{2 k} q^{p-k}\right)+b_{1,1}^{p-1} x_{n}^{2 p-2} q,
\end{aligned}
$$

with $a=\frac{2 b_{1,2}-b_{1,1}}{2 b_{1,1}} \in \mathbb{F}$, is a relation of degree $2 p$ satisfied by these polynomials. I postpone the unpleasant and lengthy verification of this relation to the appendix. The argument used in Case 1 then shows that $A=\mathbb{F}[\mathrm{V}]^{\mathbb{Z} / p}$.

In the dual case define

$$
A:=\mathbb{F}\left[x_{1}, \ldots, x_{n-2}, q^{\prime}, c_{p}^{\prime}\left(x_{n-1}\right), c_{p}^{\prime}\left(x_{n}\right)\right] /\left(r_{2 p}^{\prime}\right),
$$

where $c_{p}^{\prime}\left(x_{n-1}\right)$, resp. $c_{p}^{\prime}\left(x_{n}\right)$, are the top orbit Chern classes of $x_{n-1}$, resp. $x_{n}$,

$$
q^{\prime}=2 x_{n} f_{1}-x_{n-1} l^{\prime}-x_{n-1}^{2}
$$

is of degree 2, where $l^{\prime}=2 f_{2}-f_{1}$ is of degree 1 and $f_{1}, f_{2}$ are defined as in Case 1 , and

$$
\begin{aligned}
r_{2 p}^{\prime}= & 2 c_{p}^{\prime}(n) f_{1}^{p}-q^{\prime p}-\left(c_{p}\left(x_{n-1}\right)\right)^{2}-c_{p}\left(x_{n-1}\right) l^{\prime p}+c_{p}\left(x_{n-1}\right) l^{\prime} f_{1}^{p-1}+f_{1}^{2 p-2} q^{\prime} \\
& -\sum_{k=1}^{p-1}\left(q^{\prime p-k}(-1)^{k}\left(\frac{l^{\prime}}{2}\right)^{2 k}\right)-\sum_{k=(p-1) / 2}^{p-1} q^{\prime p-k}\left(\frac{l^{\prime}}{2}\right)^{2 k-(p-1)}\left(\frac{p-1}{2}\right)^{-1} f_{1}^{p-1}
\end{aligned}
$$

is a relation satisfied by these polynomials. I leave the verification of this relation also to the appendix. Again $A=\mathbb{F}\left[\mathrm{V}^{*}\right]^{\mathbb{Z} / p}$ is obtained in the same way as above.

For a cyclic $p$-group $G \operatorname{codim}_{\mathbb{F}}\left(\mathrm{V}^{G}\right)=\operatorname{codim}_{\mathbb{F}}\left(\mathrm{V}_{G}\right)$, so dualizing this proof leads to:

Theorem 2.3. Suppose $\mathbb{F}$ is a field of characteristic $p$ and $\mathrm{V}=\mathbb{F}^{n}$. Let $\rho: G=$ $\mathbb{Z} / p \hookrightarrow \mathrm{GL}(n, \mathbb{F})$ be a faithful representation of a cyclic group of prime order $p$, let $\operatorname{codim}_{\mathbb{F}}\left(\mathrm{V}_{G}\right)=2$. Then the ring of polynomial invariants is a complete intersection, and

$$
\mathbb{F}\left[\mathrm{V}^{*}\right]^{\mathbb{Z} / p}=\mathbb{F}\left[l_{1}, \ldots, l_{n-2}, q, c_{p, 1}, c_{p, 2}\right] /\left(r_{2 p}\right),
$$

where $\operatorname{deg}\left(l_{i}\right)=1, i=1, \ldots, n-2, \operatorname{deg}(q)=2, \operatorname{deg}\left(c_{p, j}\right)=p, j=1,2, \operatorname{deg}\left(r_{2 p}\right)=$ $2 p$. Moreover

$$
\mathbb{F}[\mathrm{V}]^{\mathbb{Z} / p}=\mathbb{F}\left[x_{1}, \ldots, x_{n-2}, q^{\prime}, c_{p, n-1}^{\prime}, c_{p, n}^{\prime}\right] /\left(r_{2 p}^{\prime}\right)
$$

is also a complete intersection, where $\operatorname{deg}\left(q^{\prime}\right)=2, \operatorname{deg}\left(c_{p, j}^{\prime}\right)=p, j=n-1, n$, $\operatorname{deg}\left(r_{2 p}^{\prime}\right)=2 p$.

\section{Corollaries}

I am going to derive some corollaries of the above theorems for more general groups.

Corollary 3.1. Let $G=\mathbb{Z} / p^{s}$ be a cyclic group of order $p^{s}$ with $\operatorname{codim}_{\mathbb{F}}\left(\mathrm{V}^{\mathbb{Z} / p^{s}}\right)=$ 2 or $\operatorname{codim}_{\mathbb{F}}\left(\mathrm{V}_{\mathbb{Z} / p^{s}}\right)=2$. Then the rings of polynomial invariants $\mathbb{F}[\mathrm{V}]^{\mathbb{Z} / p^{s}}$ and $\mathbb{F}\left[\mathrm{V}^{*}\right]^{\mathbb{Z} / p^{s}}$ are complete intersections. 
Proof. Since $\operatorname{codim}_{\mathbb{F}} \mathrm{V}^{\mathbb{Z} / p^{s}}=2$, the image of a generator under a representation $\rho$ has the form

$$
T=\left(\begin{array}{cc}
I & B \\
0 & M
\end{array}\right),
$$

with the notation as above. The $2 \times 2$-matrix $M$ has prime power order, hence $M \in \operatorname{Syl}_{p}(\mathrm{GL}(2, \mathbb{F}))$. Since this Sylow-subgroup is elementary abelian, $M$ has order $p$. Moreover matrix multiplication shows that $T$ has order $p$, if $p$ is odd. Therefore only the case $s=1$ can occur for odd primes $p$. The rings of invariants are complete intersections and the explicit structure is given by the above theorem.

If $p=2$, then $s \leq 2$. For $s=1$ again the theorems above apply. For $s=2$, $M \neq$ id and assume without loss of generality that $b_{1,1}=1$ (with the same notation as in Case 2 above),

$$
A:=\mathbb{F}\left[l_{2}, \ldots, l_{n-2}, x_{n}, c, c_{p^{2}}\left(x_{1}\right), c_{p}\left(x_{n-1}\right)\right] /\left(\bar{r}_{3 p}\right),
$$

where

$$
c_{p^{2}}\left(x_{1}\right)=x_{1}^{4}+x_{1}^{2}\left(x_{n-1} x_{n}+x_{n}^{2}+x_{n-1}^{2}\right)+x_{1}\left(x_{n-1}^{2} x_{n}+x_{n-1} x_{n}^{2}\right),
$$

resp. $c_{p}\left(x_{n-1}\right)=x_{n-1}^{2}+x_{n-1} x_{n}$, are the top orbit Chern classes of $x_{1}$, resp. $x_{n-1}$,

$$
c=x_{n-1}^{3}+x_{1}^{2} x_{n}+x_{1} x_{n}^{2}+x_{n-1} x_{n}^{2}
$$

is a generator of degree $3, l_{i}, i=2, \ldots, n-2$, are defined in Theorem 2.1, Case 2, and

$$
\bar{r}_{3 p}=c_{p^{2}}\left(x_{1}\right) x_{n}^{2}+c_{p}^{3}\left(x_{n-1}\right)+c^{2}+c c_{p}\left(x_{n-1}\right) x_{n},
$$

is a relation of degree $3 p=6$ satisfied by these polynomials. (This is an easy, but long, calculation.) $A \subset \mathbb{F}[\mathrm{V}]^{\mathbb{Z} / 4}$ is obtained in the same way as above. Using the prime elements $x_{n}, c_{p}\left(x_{n-1}\right), l_{i}$, for $i=2, \ldots, n-2$, as in the argument above shows that $A=\mathbb{F}[\mathrm{V}]^{\mathbb{Z} / 4}$.

In the dual case define

$$
A:=\mathbb{F}\left[x_{1}, \ldots, x_{n-2}, c^{\prime}, c_{p}^{\prime}\left(x_{n-1}\right), c_{p^{2}}^{\prime}\left(x_{n}\right)\right] /\left(\bar{r}_{3 p}^{\prime}\right),
$$

where $c_{p}^{\prime}\left(x_{n-1}\right)=x_{n-1}^{2}+x_{n-1} f_{1}$, resp.

$$
\begin{aligned}
c_{p^{2}}^{\prime}\left(x_{n}\right)= & x_{n}^{4}+x_{n}^{2}\left(x_{n-1}^{2}+f_{2}^{2}+x_{n-1} f_{1}+f_{1} f_{2}+f_{1}^{2}\right) \\
& +x_{n}\left(f_{1} x_{n-1}^{2}+f_{1} f_{2}^{2}+f_{1}^{2} x_{n-1}+f_{1}^{2} f_{2}\right),
\end{aligned}
$$

are the top orbit Chern classes of $x_{n-1}$, resp. $x_{n}$,

$$
c^{\prime}=x_{n-1}^{3}+x_{n-1} f_{2}^{2}+x_{n-1}^{2} f_{1}+x_{n-1} f_{1} f_{2}+x_{n} f_{1}^{2}+x_{n}^{2} f_{1},
$$

is a generator of degree $3, f_{1}$ and $f_{2}$ as in the dual of Case 2 of Theorem 2.1, and

$$
\begin{aligned}
\bar{r}_{3 p}^{\prime}= & c_{p^{2}}^{\prime}\left(x_{n}\right) f_{1}^{2}+c_{p}^{\prime 3}\left(x_{n-1}\right)+c^{\prime 2}+c^{\prime} f_{1} c_{p}^{\prime}\left(x_{n-1}\right) \\
& +f_{2} f_{1}\left(f_{1}+f_{2}\right) c^{\prime}+f_{2}^{2}\left(f_{1}+f_{2}\right)^{2} c_{p}^{\prime}\left(x_{n-1}\right)
\end{aligned}
$$

is a relation satisfied by these polynomials. Again this is a lengthy but easy calculation, $A \subset \mathbb{F}\left[\mathrm{V}^{*}\right]^{G}$ can be shown as above and equality is obtained in the same way as above by inverting the linear generators and $c_{p}\left(x_{n-1}\right)$, and using Nagata's theorem.

Dualizing leads to the corresponding results for $\operatorname{codim}_{\mathbb{F}} \mathrm{V}_{G}=2$.

The following corollary is a straightforward generalization for representations of block form. 
Corollary 3.2. Let $G$ be an abelian p-group of order $p^{s}$. Suppose

$$
G=\bigoplus_{i=1}^{\beta} G_{i}
$$

is a direct sum decomposition, and $x_{1}, \ldots, x_{n}$ is a basis of the dual vector space $\mathrm{V}^{*}$, which decomposes $\mathrm{V}^{*}$ to a direct sum of submodules

$$
\mathrm{V}^{*}=\left(\bigoplus_{i=1}^{\beta} \mathrm{V}^{*}{ }_{i}\right) \oplus\left(\bigoplus_{i=\beta+1}^{n} \mathrm{~V}^{*}{ }_{i}\right) .
$$

If $G_{i}$ acts on $\mathrm{V}^{*}{ }_{i}$ and trivial on $V^{*}{ }_{i^{\prime}}$ for $i \neq i^{\prime}$ and $\operatorname{codim}_{\mathbb{F}}\left(\mathrm{V}_{i}^{G_{i}}\right) \leq 2$, then the ring of polynomial invariants is a complete intersection. The explicit formulae for generators and relations may be derived from the theorems above.

Since complete intersection rings are Cohen-Macaulay, and the ring of polynomial invariants of any group $G$ is Cohen-Macaulay when the ring of invariants of its $p$-Sylow subgroup is, I get the following corollary, which is in part a result of Ellingsrud-Skjelbred [2]:

Corollary 3.3. Let $G \hookrightarrow \mathrm{GL}(n, \mathbb{F})$ be a faithful representation of any group whose order is not prime to $p$, the characteristic of the field $\mathbb{F}$. If the $p$-Sylow subgroup of $G$ has the form described in the above Corollary 3.2, then the ring of polynomial invariants of $G, \mathbb{F}[\mathrm{V}]^{G}$ and the ring of invariants of the dual representation, $\mathbb{F}\left[\mathrm{V}^{*}\right]^{G}$, are Cohen-Macaulay.

\section{APPENDiX}

Recalling the notation of Theorem 2.1 I am going to verify the relations $r_{2 p}$ and $r_{2 p}^{\prime}$ mentioned in Case 2 of the proof. ${ }^{6}$

Lemma A1. Consider an orbit of the form $a+i b$, for $0 \leq i \leq p-1$. Then the Chern classes $c_{k}, k=1, \ldots, p$, are given by

$$
c_{k}= \begin{cases}0 & \text { for } k<p-1, \\ -b^{p-1} & \text { for } k=p-1, \\ a^{p}-a b^{p-1} & \text { for } k=p .\end{cases}
$$

Proof. The formula for the top orbit Chern class $c_{p}$ is well known. The reader might be convinced after noticing that the polynomial $a^{p}-a b^{p-1}$ has the right degree and the same zeros as $c_{p}:=\prod_{i=0}^{p-1}(a+i b)$. If $k \leq p-1$, then Newton's formula [7], p. 2 , expresses the orbit Chern classes $c_{k}$ in terms of the orbit power sums, since they are just the elementary symmetric functions in the orbit elements:

$$
k c_{k}=\sum_{j=1}^{k}(-1)^{j-1} P_{j} c_{k-j},
$$

where $c_{0}:=1$. Then

$$
P_{j}=\sum_{i=0}^{p-1}(a+i b)^{j}=\sum_{\alpha=0}^{j}\left(\begin{array}{c}
j \\
\alpha
\end{array}\right) a^{j-\alpha} b^{\alpha} \sum_{i=0}^{p-1} i^{\alpha} .
$$

\footnotetext{
${ }^{6}$ It ought to be possible to do the following calculations with the help of a computer algebra program.
} 
By von Staudt's theorem [3], p. 91,

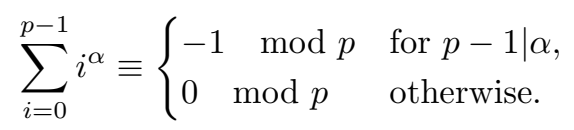

Therefore

$$
P_{j}= \begin{cases}-b^{p-1} & \text { for } j=p-1 \\ 0 & \text { otherwise }\end{cases}
$$

and hence

$$
c_{k}= \begin{cases}0 & \text { for } k<p-1 \\ -b^{p-1} & \text { for } k=p-1\end{cases}
$$

as required.

Lemma A2. Consider an orbit of the form $a+i b+i^{2} c$, for $0 \leq i \leq p-1$. Then for $p \neq 2$ the Chern classes $c_{k}, k=1, \ldots, p-1$, are given by

$$
k c_{k}= \begin{cases}0 & \text { for } k<\frac{p-1}{2} \\ (-1)^{k-1} P_{k} & \text { for } \frac{p-1}{2} \leq k<p-1 \\ \left(\frac{p-1}{2}\right)^{-1} P_{\frac{p-1}{2}}^{2}-P_{p-1} & \text { for } k=p-1\end{cases}
$$

where $P_{k}:=\sum_{i=0}^{p-1}\left(a+i b+i^{2} c\right)^{k}$ are the orbit power sums. For $p=2$ they are

$$
c_{k}= \begin{cases}b+c & \text { for } k=1, \\ a^{2}+a(b+c) & \text { for } k=2 .\end{cases}
$$

Proof. The formulae for $p=2$ are routine. The case $p \neq 2$ can be obtained in the same way as Lemma A1 by computing the orbit power sums with the help of von Staudt's theorem and using Newton's formula to get the orbit Chern classes.

Lemma A3. Consider an orbit of the form $(\tilde{a}+i \tilde{c})^{2}$, for $i=0, \ldots, p-1$. Then the orbit Chern classes are given by

$$
c_{k}= \begin{cases}0 & \text { for } k \neq \frac{p-1}{2}, k \neq p-1 \text { and } k \neq p, \\ (-1)^{k} \frac{1}{k} \tilde{c}^{2 k} & \text { for } k=\frac{p-1}{2} \text { or } k=p-1, \\ \left(\tilde{a}^{p}-\tilde{a} \tilde{c}^{p-1}\right)^{2} & \text { for } k=p,\end{cases}
$$

for $p \neq 2$. For $p=2$ they are

$$
c_{k}= \begin{cases}\tilde{c}^{2} & \text { for } k=1, \\ \tilde{a}^{2}\left(\tilde{a}^{2}+\tilde{c}^{2}\right) & \text { for } k=2 .\end{cases}
$$

Proof. Again the formulae for $p=2$ are obvious. The case $p \neq 2$ can be obtained by Lemma A2 using once more von Staudt's theorem to compute explicitly the remaining orbit power sums. The top orbit Chern class can be derived by Lemma A1. 
Lemma A4. With the notation of Theorem 2.1

$$
\begin{aligned}
r_{2 p}= & 2 c_{p}\left(x_{1}\right) x_{n}^{p}-q^{p}-b_{1,1} c_{p}\left(x_{n-1}\right)^{2}-\sum_{k=1}^{p-1}\left((-1)^{k} b_{1,1}^{k} a^{2 k} x_{n}^{2 k} q^{p-k}\right) \\
& -\sum_{k=(p-1) / 2}^{p-1} b_{1,1}^{k}\left((-1)^{k}\left(\frac{p-1}{2}\right)^{-1} a^{2 k-(p-1)} x_{n}^{2 k} q^{p-k}\right)+b_{1,1}^{p-1} x_{n}^{2 p-2} q,
\end{aligned}
$$

where $a=\frac{2 b_{1,2}-b_{1,1}}{2 b_{1,1}} \in \mathbb{F}$. Recall that $b_{1,1} \neq 0$ and $p \neq 2$.

Proof. Observe that

$$
\begin{aligned}
2 c_{p}\left(x_{1}\right) x_{n}^{p} & =c_{p}\left(q+b_{1,1} x_{n-1}^{2}+\left(2 b_{1,2}-b_{1,1}\right) x_{n-1} x_{n}\right) \\
& =q^{p}+\sum_{k=1}^{p} q^{p-k} c_{k}\left(b_{1,1} x_{n-1}^{2}+\left(2 b_{1,2}-b_{1,1}\right) x_{n-1} x_{n}\right),
\end{aligned}
$$

where the last equality holds, because $q$ is invariant and $c_{0}:=1$. The next step is to evaluate the orbit Chern classes $c_{k}\left(b_{1,1} x_{n-1}^{2}+\left(2 b_{1,2}-b_{1,1}\right) x_{n-1} x_{n}\right), k=1, \ldots, p$. Since the top orbit Chern class is multiplicative one gets from Lemma A1

$$
\begin{aligned}
& c_{p}\left(b_{1,1} x_{n-1}^{2}+\left(2 b_{1,2}-b_{1,1}\right) x_{n-1} x_{n}\right) \\
& \quad=c_{p}\left(x_{n-1}\right) c_{p}\left(b_{1,1} x_{n-1}+\left(2 b_{1,2}-b_{1,1}\right) x_{n}\right)=b_{1,1} c_{p}\left(x_{n-1}\right)^{2} .
\end{aligned}
$$

It remains to compute the other Chern classes $c_{k}\left(b_{1,1} x_{n-1}^{2}+\left(2 b_{1,2}-b_{1,1}\right) x_{n-1} x_{n}\right)$ for $k=1, \ldots, p-1$. In order to apply Lemma A3 note that

$$
\begin{aligned}
& b_{1,1} x_{n-1}^{2}+\left(2 b_{1,2}-b_{1,1}\right) x_{n-1} x_{n} \\
& \quad=b_{1,1}\left(x_{n-1}^{2}+\frac{2 b_{1,2}-b_{1,1}}{b_{1,1}} x_{n-1} x_{n}+a^{2} x_{n}^{2}-a^{2} x_{n}^{2}\right),
\end{aligned}
$$

where $a=\frac{2 b_{1,2}-b_{1,1}}{2 b_{1,1}}$. Since $a^{2} x_{n}^{2}$ is invariant, the Chern classes for $k=1, \ldots, p-1$ are

$$
\begin{aligned}
c_{k}( & \left.b_{1,1} x_{n-1}^{2}+\left(2 b_{1,2}-b_{1,1}\right) x_{n-1} x_{n}\right) \\
= & b_{1,1}^{k}\left(\sum_{i=0}^{k}(-1)^{k-i} a^{2(k-i)} x_{n}^{2(k-i) i} c_{i}\left(\left(x_{n-1}+a x_{n}\right)^{2}\right)\right) \\
= & \begin{cases}(-1)^{k} b_{1,1}^{k} a^{2 k} x_{n}^{2 k} & \text { for } k<\frac{p-1}{2}, \\
b_{1,1}^{k}\left((-1)^{k} a^{2 k} x_{n}^{2 k}+c_{k}\left(\left(x_{n-1}+a x_{n}\right)^{2}\right)\right) & \text { for } k=\frac{p-1}{2}, \\
b_{1,1}^{k}\left((-1)^{k} a^{2 k} x_{n}^{2 k}+(-1)^{k-\frac{p-1}{2}} a^{2 k-(p-1)} x_{n}^{2 k-(p-1)} c_{\left.\frac{p-1}{2}\left(\left(x_{n-1}+a x_{n}\right)^{2}\right)\right)}\right. & \text { for } \frac{p-1}{2}<k<p-1, \\
b_{1,1}^{k}\left((-1)^{k} a^{2 k} x_{n}^{2 k}+(-1)^{\frac{p-1}{2}} a^{p-1} x_{n}^{p-1} c_{p-1}\left(\left(x_{n-1}+a x_{n}\right)^{2}\right)\right. \\
+c_{p-1}\left(\left(x_{n-1}+a x_{n}\right)^{2}\right) & \text { for } k=p-1, \\
=(-1)^{k} b_{1,1}^{k} a^{2 k} x_{n}^{2 k}+ \begin{cases}0 & \text { for } k<\frac{p-1}{2}, \\
(-1)^{\frac{p-1}{2}}\left(\frac{p-1}{2}\right)^{-1} b_{1,1}^{k} x_{n}^{p-1} & \text { for } k=\frac{p-1}{2}, \\
(-1)^{k} a^{2 k-(p-1)} b_{1,1}^{k} x_{n}^{2 k}\left(\frac{p-1}{2}\right)^{-1} & \text { for } \frac{p-1}{2}<k<p-1, \\
a^{p-1} b_{1,1}^{p-1} x_{n}^{2 p-2}\left(\frac{p-1}{2}\right)^{-1}-b_{1,1}^{p-1} x_{n}^{2 p-2} & \text { for } k=p-1,\end{cases} \end{cases}
\end{aligned}
$$


where the last two equalities follow from Lemma A2, resp. A3. Combining these results leads to the desired formula.

In the same way one proves

Lemma A5. With the notation of Theorem 2.1

$$
\begin{aligned}
r_{2 p}^{\prime}= & 2 c_{p}^{\prime}\left(x_{n}\right) f_{1}^{p}-q^{\prime p}-\left(c_{p}\left(x_{n-1}\right)\right)^{2}-c_{p}\left(x_{n-1}\right) l^{\prime p}+c_{p}\left(x_{n-1}\right) l^{\prime} f_{1}^{p-1}+f_{1}^{2 p-2} q^{\prime} \\
& -\sum_{k=1}^{p-1}\left(q^{\prime p-k}(-1)^{k}\left(\frac{l^{\prime}}{2}\right)^{2 k}\right)-\sum_{k=(p-1) / 2}^{p-1} q^{\prime p-k}\left(\frac{l^{\prime}}{2}\right)^{2 k-(p-1)}\left(\frac{p-1}{2}\right)^{-1} f_{1}^{p-1},
\end{aligned}
$$

where as above $l^{\prime}=2 f_{2}-f_{1}$ is of degree 1 , and $p \neq 2$.

\section{REFERENCES}

1. D. Eisenbud, Commutative algebra, Graduate Texts in Mathematics, vol. 150, Springer Verlag, New York and Berlin, 1995. CMP 95:10

2. G. Ellingsrud and T. Skjelbred, Profondeur d'anneaux d'invariants en caractéristique $p$, Comp. Math. 41 (1980), 233-244. MR 82c:13015

3. G. H. Hardy and E. M. Wright, An introduction to the theory of numbers, 4th edition, Clarendon Press, Oxford, 1960.

4. P. S. Landweber and R. E. Stong, The depth of rings of invariants over finite fields, Proc. New York Number Theory Seminar, 1984, Lecture Notes in Math., vol. 1240, Springer Verlag, New York, 1987. MR 88k:13004

5. M. Nagata, A remark on the unique factorization theorem, J. Math. Soc. Japan 9 (1957), 143-145. MR 18:869a

6. H. Nakajima, Invariants of finite abelian groups generated by transvections, Tokyo J. Math. 3 (1980), 201-214. MR 82e:14058

7. L. Smith, Polynomial invariants of finite groups, A. K. Peters LTD, Wellesley, MA, 1995. CMP 95:11

Institut für Algebra und Geometrie, Otto-von-Guericke-Universität, Postfach 4120, D 39016 Magdeburg, Germany

E-mail address: mara.neusel@mathematik.uni-magdeburg.de 